\title{
ON THE MONOTONE CONVERGENCE OF SOME ITERATIVE PROCEDURES IN PARTIALLY ORDERED BANACH SPACES
}

\author{
IOANNIS K. ARGYROS
}

\begin{abstract}
We provide some enclosure methods for the solution of a nonlinear equation in a partially ordered Banach space. By using a certain projection operator we show that the solution can be obtained from the solution of a system of linear algebraic equations.
\end{abstract}

\section{Introduction.}

In this paper we study the convergence of the iterative procedures

$$
F\left(y_{n}\right)+P A\left(y_{n}, y_{n-1}\right)\left(y_{n+1}-y_{n}\right)=0
$$

and

$$
F\left(x_{n}\right)+P A\left(y_{n}, y_{n-1}\right)\left(x_{n+1}-x_{n}\right)=0
$$

to a zero $z$ of the nonlinear equation

$$
F(x)=0
$$

Here, $F$ is a nonlinear operator defined on a convex subset $D$ of a Banach space $E$ with values in a Banach space $\hat{E}$. For fixed $x, y \in D, A(x, y)$ denotes a linear operator from $E$ to $\hat{E}$ and $P$ is a projection operator $\left(P^{2}=P\right)$, that projects the space $\hat{E}$ into $\hat{E}_{p} \subseteq \hat{E}$.

The study of iterative procedures under partial ordering started in 1939 by $\mathrm{L}$.V. Kantorovich [4]. In 1952, A. Baluev [3] gave sufficient conditions for the monotone convergence of Newton's method in partially ordered topological spaces. In 1970, J.W. Schmidt and H. Leonhardt [7] used the Secant method for constructing pairs of monotone sequences converging to a zero of $F$. Various generalizations were later obtained by many authors, when $p=I$, the identity operator on $F$ [3], [4], [5], [6], [7], [8]. In this case the iterates $x_{n}$ and $y_{n}, n \geq 0$ generated by (1) and (2) can rarely be computed in infinite

Received August 14, 1989.

Key words and phrases: Monotone convergence, Banach space.

(1980) A.M.S. classification codes: 47D15, 47H17, 65. 
dimensional spaces. However, if $E_{p}$ is finite dimensional with $\operatorname{dim}\left(E_{p}\right)=N$, then from (1) and (2) we obtain systems of linear algebraic equations of order at most $N$.

We provide sufficient conditions for the monotone convergence of the sequence $\left\{x_{n}\right\}$ and $\left\{y_{n}\right\}$ to a zero $z$ of $F$, as well as error bounds on the distances $\left\|x_{n}-z\right\|,\left\|x_{n}-x_{n-1}\right\|$, $\left\|y_{n}-z\right\|$ and $\left\|y_{n}-y_{n-1}\right\|$.

\section{Preliminaries.}

In this section we reproduce some definitions from the theory of partially ordered linear space [4], [6].

Let $E$ be a linear space. A subset $K$ of $E$ is called a cone if $K+K \subset K$ and $\alpha K \subset K$ for $\alpha>0$. The cone $K$ is proper if $K \cap\{-K\}=\{0\}$. The relations " $\leq$ " defined by

$$
x \leq y \text { if and only if } y-x \in K
$$

is a partial ordering on $K$ which is compatible with the linear structure of this space. Two elements $x$ and $y$ of $E$ are called comparable if either $x \leq y$ or $y \leq x$ holds. The space $E$ endowed with the above relation is called a partially ordered linear space (POL-space). If $E$ has a topology compatible with its linear structure and if the cone $K$ is closed in that topology then $E$ is called a partially ordered topological space (POTL-space).

We remark that in a POTL-space the intervals $[a, b]=\{x ; a \leq x \leq b\}$ are closed sets. A lot of examples show that the closedness of the nonnegative cone is not, in general, a strong enough connection between the ordering and the topology [8]. A stronger connection is considered by the following definitions.

Definition 1. A POTL-space is called normal if given a local base $\mathcal{U}$ for the topology, there exists a positive number $\eta$ so that if $0 \leq z \in U \in \mathcal{U}$, then $[0, z] \subset \eta U$.

Definition 2. A POTL-space is called regular if every order bounded increasing sequence has a limit.

If the topology of a POTL-space is given by a norm then this space is called a partially ordered normed space ( $\mathrm{PON}$-space). If a $\mathrm{PON}$-space is complete with respect to its topology then it is called a partially ordered Banach space (POB-space). According to Definition 1 a PON-space is normal if and only if there exists a positive number $\alpha$ such that

$$
\|x\| \leq \alpha\|y\| \quad \text { for all } \quad x, y \in E \text { with } 0 \leq x \leq y .
$$

Note that any regular POB-space is normal. The reverse is not true. For example, the space $C[0,1]$ of all continuous real functions defined on $[0,1]$, ordered by the cone of nonnegative functions, is normal but is not regular. All finite dimensional POL-spaces are both normal and regular.

Let us define now some special type of operators acting between two POL-spaces. If $E$ and $\hat{E}$ are two linear spaces then we denote by $(E, \hat{E})$ the set of all operators 
from $E$ into $\hat{E}$ and by $L(E, \hat{E})$ the set of all linear operators from $E$ into $\hat{E}$. If $E$ and $\hat{E}$ are topological linear space then we denote by $B(E, \hat{E})$ the set of all continuous linear operators from $E$ into $\hat{E}$. Now let $E$ and $\hat{E}$ be two POL-spaces and consider an operator $G \in(E, \hat{E}) . G$ is called isotone (resp. antitone) if $x \leq y$ implies $G(x) \leq G(y)$ (resp. $G(x) \geq G(y)$ ). $G$ is called nonnegative if $G(x) \geq 0$ implies $x \geq 0$. For linear operators the nonnegativity is clearly equivalent with the isotony. Also, a linear operator is inverse nonnegative if and only if it is invertible and its inverse is nonnegative. If $G$ is a nonnegative operator then we write $G \geq 0$. If $G$ and $H$ are two operators from $E$ into $\hat{E}$ such that $H-G$ is nonnegative then we write $G \geq H$. If $Z$ is a linear space then we denote by $I=I_{z}$ the identity operator in $Z$. If $Z$ is a POL-space then we obviously have $I \geq 0$. Suppose $E$ and $\hat{E}$ are two POL-space and consider the operators $T \in L(E, \hat{E})$ and $S \in L(E, \hat{E})$. If $S T \leq I_{E}$ (resp. if $S T \geq I_{E}$ ) then $S$ is called a left subinverse (resp. superinverse) of $T$ and $T$ is called a right subinverse (resp. superinverse) of $S$. We say that $S$ is a subinverse of $T$ if $S$ is a left as well as right subinverse of $T$.

\section{Monotone convergence results.}

We can now formulate the main theorem.

Theorem 1. Let $E$ be a regular POTL-space, $\hat{E}$ a POTL-space and $F: D \subset E \longrightarrow$ $\hat{E}$.

Assume

(i) there exist points $x_{0}, y_{0}, y_{-1}$ in $D$ with

$$
x_{0} \leq y_{0} \leq y_{1},\left[x_{0}, y_{-1}\right] \subset D, F\left(x_{0}\right) \leq 0 \leq F\left(y_{0}\right) .
$$

(ii) Set

$$
\begin{gathered}
Q_{1}=\left\{(x, y) \in E^{2} ; x_{0} \leq x \leq y \leq y_{0}\right\}, \\
Q_{2}=\left\{\left(y, y_{-1}\right) \in E^{2} ; x_{0} \leq y \leq y_{0}\right\},
\end{gathered}
$$

and

$$
Q_{3}=Q_{1} \cup Q_{2} .
$$

Let $A: Q_{3} \longrightarrow B(E, \hat{E})$ be an operator such that

$$
P A(w, z)(y-x) \leq F(y)-F(x)
$$

for all $(x, y),(y, w) \in Q_{1},(w, z) \in Q_{3}$.

(iii) The linear operator $P A(u, v)$ has a continuous nonsingular nonnegative left subinverse.

Then there exist two sequences $\left\{x_{n}\right\},\left\{y_{n}\right\}, n \geq 1$ and two points $x^{*}, y^{*}$ of $E$ such that for all $n \geq 0$

$$
F\left(y_{n}\right)+P A\left(y_{n}, y_{n-1}\right)\left(y_{n+1}-y_{n}\right)=0
$$




$$
\begin{gathered}
F\left(x_{n}\right)+P A\left(y_{n}, y_{n-1}\right)\left(x_{n+1}-x_{n}\right)=0, \\
F\left(x_{n}\right) \leq 0 \leq F\left(y_{n}\right), \\
x_{0} \leq x_{1} \leq \ldots \leq x_{n} \leq x_{n+1} \leq y_{n+1} \leq y_{n} \leq \cdots \leq y_{1} \leq y_{0}, \\
\lim _{n \rightarrow \infty} x_{n}=x^{*} \quad \text { and } \quad \lim _{n \rightarrow \infty} y_{n}=y^{*} .
\end{gathered}
$$

Moreover, if the operator $L_{n}=P A\left(y_{n}, y_{n-1}\right)$ are inverse nonnegative then any zero $z$ of $F$ in $\left[x_{0}, y_{0}\right]$ is in $\left[x^{*}, y^{*}\right]$.

Proof. Let $\bar{L}_{0}$ be a continuous nonsingular nonnegative left subinverse of $L_{0}$. Let us define the operator

$$
T_{1}:\left[0, y_{0}-x_{0}\right] \longrightarrow E
$$

by

$$
T_{1}(x)=x-\bar{L}_{0}\left(F\left(x_{0}\right)+L_{0}(x)\right) .
$$

Then $T_{1}$ is clearly isotone, continuous with

$$
T_{1}(0)=-\bar{L}_{0} F\left(x_{0}\right) \geq 0
$$

and

$$
\begin{aligned}
T_{1}\left(y_{0}-x_{0}\right) & =y_{0}-x_{0}-\bar{L}_{0} F\left(y_{0}\right)+\bar{L}_{0}\left(F\left(y_{0}\right)-F\left(x_{0}\right)-L_{0}\left(y_{0}-x_{0}\right)\right) \\
& \leq y_{0}-x_{0}-\bar{L}_{0} F\left(y_{0}\right) \leq y_{0}-x_{0} .
\end{aligned}
$$

By the well-known Kantorovich theorem on nonlinear equations on partially ordered space [4], we deduce the existence of a fixed point $v$ of $T_{1}$ in $\left[0, y_{0}-x_{0}\right]$. Set $x_{1}=x_{0}+v$, then

$$
F\left(x_{0}\right)+L_{0}\left(x_{1}-x_{0}\right)=0, \quad x_{0} \leq x_{1} \leq y_{0}
$$

By (7), we get

$$
F\left(x_{1}\right)=F\left(x_{1}\right)-F\left(x_{0}\right)+L_{0}\left(x_{0}-x_{1}\right) \leq 0 .
$$

Similarly, let us consider the operator $T_{2}:\left[0, y_{0}-x_{1}\right] \longrightarrow E$ given by

$$
T_{2}(x)=x+\bar{L}_{0}\left(F\left(y_{0}\right)-L_{0}(x)\right)
$$

The operator $T_{2}$ is isotone, continuous with

$$
T_{2}(0)=\bar{L}_{0} F\left(y_{0}\right) \geq 0
$$

and

$$
\begin{aligned}
T_{2}\left(y_{0}-x_{1}\right) & =y_{0}-x_{1}-\bar{L}_{0} F\left(x_{1}\right)+\bar{L}_{0}\left(F\left(y_{0}\right)-F\left(x_{1}\right)-L_{0}\left(y_{0}-x_{1}\right)\right) \\
& \leq y_{0}-x_{1}-\bar{L}_{0} F\left(x_{1}\right) \leq y_{0}-x_{1} .
\end{aligned}
$$


As before, we deduce the existence of a fixed point $v_{1}$ in $\left[0, y_{0}-x_{1}\right]$ of $T_{2}$. Set $y_{1}=y_{0}-v_{1}$ to obtain

$$
F\left(y_{0}\right)+L_{0}\left(y_{1}-y_{0}\right)=0, \quad x_{1} \leq y_{1} \leq y_{0}
$$

By (7), we get

$$
F\left(y_{1}\right)=F\left(y_{1}\right)-F\left(y_{0}\right)-L_{0}\left(y_{1}-y_{0}\right) \geq 0 \text {. }
$$

Using induction on $n$, we generate two sequences $\left\{x_{n}\right\}$ and $\left\{y_{n}\right\}, n \geq 1$ satisfying (1), (2), (8), (9). But the space $E$ is regular, therefore there exist $x^{*}, y^{*} \in E$ satisfying (10), with $x^{*} \leq y^{*}$.

Let $z$ be a zero of $F$ in $\left[x_{0}, y_{0}\right]$. Then, we have

$$
L_{0}\left(y_{1}-z\right)=L_{0}\left(y_{0}\right)-F\left(y_{0}\right)-L_{0}(z)=L_{0}\left(y_{0}-z\right)-\left(F\left(y_{0}\right)-F(z)\right) \geq 0
$$

and

$$
L_{0}\left(x_{1}-z\right)=L_{0}\left(x_{0}\right)-F\left(x_{0}\right)-L_{0}(z)=L_{0}\left(x_{0}-z\right)-\left(F\left(x_{0}\right)-F(z)\right) \leq 0 .
$$

If the operator $L_{0}$ is inverse isotone, then $x_{1} \leq z<y_{1}$. Similarly, using induction on $n$, we get $x_{n} \leq z<y_{n}$. That is, $x^{*} \leq z \leq y^{*}$.

That completes the proof of the theorem.

We now show that if certain conditions are satisfied then $x^{*}$ and $y^{*}$ are zeros of $F$.

Theorem 2. Suppose that the operator $F$ is continuous at $x^{*}, y^{*}$ and the hypotheses of Theorem 1 are satisfied. Moreover, assume that one of the following conditions is satisfied

(a) $x^{*}=y^{*}$;

(b) $E$ is normal and there exists an operator $T: E \longrightarrow \hat{E}$ with $T(0)=0$ which has an isotone inverse continuous at the origin and such that $L_{n} \leq T$ for sufficiently large $n$;

(c) $\hat{E}$ is normal and there exists an operator $S: E \longrightarrow \hat{E}$ with $S(0)=0$ continuous at the origin and such that $L_{n} \leq S$ for sufficiently large $n$; and

(d) the operator $L_{n}, n \geq 0$ are equicontinuous.

Then

$$
F\left(x^{*}\right)=F\left(y^{*}\right)=0
$$

Proof.

(a) By the continuity of $F$ and (8) we get $F\left(x^{*}\right) \leq 0 \leq F\left(x^{*}\right)$. That is $F\left(x^{*}\right)=0$.

(b) By (1), (2), (8)-(10) we have

$$
\begin{aligned}
& T\left(x_{n}-x_{n+1}\right) \leq L n\left(x_{n}-x_{n+1}\right)=F\left(x_{n}\right) \leq 0 \\
& 0 \leq F\left(y_{n}\right)=L_{n}\left(y_{n}-y_{n+1}\right) \leq T\left(y_{n}-y_{n+1}\right) .
\end{aligned}
$$


So,

$$
x_{n}-x_{n+1} \leq T^{-1} F\left(x_{n}\right) \leq 0, \quad 0 \leq T^{-1} F\left(y_{n}\right) \leq y_{n}-y_{n+1} .
$$

But $E$ is normal and $\lim _{n \rightarrow \infty}\left(x_{n}-x_{n+1}\right)=\lim _{n \rightarrow \infty}\left(y_{n}-y_{n+1}\right)=0$. Therefore, $\lim _{n \rightarrow \infty} T^{-1} F\left(x_{n}\right)=\lim _{n \rightarrow \infty} T^{-1} F\left(y_{n}\right)=0$. That is, $F\left(x^{*}\right)=F\left(y^{*}\right)=0$, by continuity.

(c) For sufficiently large $n$, as in (b) we get

$$
S\left(x_{n}-x_{n+1}\right) \leq F\left(x_{n}\right) \leq 0, \quad 0 \leq F\left(y_{n}\right) \leq S\left(y_{n}-y_{n+1}\right)
$$

Since $\hat{E}$ is normal and $F, S$ are continuous we get $F\left(x^{*}\right)=F\left(y^{*}\right)=0$.

(d) By the equicontinuity of the operators $L_{n}$ it follows that $\lim _{n \rightarrow \infty} L_{n}\left(v_{n}\right)=0$ if $\lim _{n \rightarrow \infty} v_{n}=0$. In particular,

$$
\lim _{n \rightarrow \infty} L_{n}\left(x_{n}-x_{n+1}\right)=\lim _{n \rightarrow \infty} L_{n}\left(y_{n}-y_{n+1}\right)=0
$$

But from (1), (2) and the continuity of $F$ at $x^{*}$ and $y^{*}$ we get $F\left(x^{*}\right)=F\left(y^{*}\right)=0$.

That completes the proof of the theorem.

We now give sufficient conditions for the uniqueness of a zero of $F$ in $\left[x_{0}, y_{0}\right]$.

Theorem 3. Let $E$ and $\hat{E}$ be two $P O L$-spaces and $F: D \subset E \longrightarrow \hat{E}$. Suppose

(a) there exist $x_{0}, y_{0} \in D$ such that $x_{0} \leq y_{0}$ and $\left[x_{0}, y_{0}\right] \subset D$. Let $Q_{1}=\{(x, y) \in$ $\left.E^{2} ; x_{0} \leq x \leq y \leq y_{0}\right\}$

(b) ther exists an operator $T: Q_{1} \longrightarrow L(E, \hat{E})$ such that $P T(x, y)$ has a nonnegative lefi superinverse for each $(x, y) \in Q_{1}$ and $F(y)-F(x) \geq P T(x, y)(y-x)$ for all $(x, y) \in Q_{1}$.

(c) $\left(x^{*}, y^{*}\right) \in Q_{1}$ and $F\left(x^{*}\right)=F\left(v^{*}\right)$.

Then

$$
x^{*}=y^{*} \text {. }
$$

Proof. Let $S\left(x^{*}, y^{*}\right)$ be a nonnegative left superinverse of $P T\left(x^{*}, y^{*}\right)$. We have

$$
0 \leq y^{*}-x^{*} \leq S\left(x^{*}, y^{*}\right) P T\left(x^{*}, y^{*}\right)\left(y^{*}-x^{*}\right) \leq S\left(X^{*}, y^{*}\right)\left(F\left(y^{*}\right)-F\left(x^{*}\right)\right)=0
$$

Hence, $x^{*}=y^{*}$.

That completes the proof of the theorem.

Note that the estimates (9) give automatic error bounds at each step of the iterative procedures (1) and (2). In order to obtain further estimates on the distances $\left\|x_{n}-x^{*}\right\|$, $\left\|x_{n+1}-x_{n}\right\|$ (similarly for $\left\|\dot{y}_{n}-y^{*}\right\|$ and $\left.\left\|y_{n+1}-y_{n}\right\|\right)$ we develop the following theorem. 
Theorem 4. Let $E$ and $\hat{E}$ be Banach spaces and $F: D \subset E \longrightarrow \hat{E}$. Assume

(a) the linear operator $P A(u, v)$ is invertible and

$$
\left\|(P A(u, v))^{-1}\right\| \leq b
$$

for all $u, v \in U\left(x_{0}, r\right)=\left\{x \in E /\left\|x-x_{0}\right\| \leq r\right\}$

(b) the following conditions are satisfied

$$
\begin{gathered}
\|F(y)-F(x)-P A(u, v)(y-x)\| \leq c\|y-x\|^{1+d_{x}}, \\
d_{x} \geq 0
\end{gathered}
$$

for $x \in U\left(x_{0}, r\right)$ and $u, v \in \bar{U}\left(x_{0}, r\right)$;

(c) the following estimates are true

$$
\begin{gathered}
\left\|F\left(x_{0}\right)\right\| \leq b_{0} \\
b b_{0}=\eta<1
\end{gathered}
$$

and

$$
h=b c \eta^{d}<1
$$

where

$$
d=\min _{n} d_{n}=\min _{n} d_{x_{n}}
$$

Then the operator $F$ has a zero $x^{*}$ in $U\left(x_{0}, r\right)$ and

$$
\begin{aligned}
\left\|x_{n}-x^{*}\right\| & \leq q_{n} \eta, \quad n \geq 0 \\
\left\|x_{n+1}-x_{n}\right\| & \leq b c\left\|x_{n}-x_{n-1}\right\|^{1+d_{n}}
\end{aligned}
$$

where

$$
\begin{gathered}
r=g_{0} \eta \\
q_{n}=\sum_{i=1}^{\infty} h^{t_{i}}, \quad n \geq 0, \\
t_{0}=0, \quad t_{1}=1
\end{gathered}
$$

and

$$
t_{n}=1+\left(1+d_{n}\right)+\cdots+\left(1+d_{2}\right)+\cdots+\left(1+d_{n}\right) .
$$

Proof. Let us assume that $x_{1}, x_{2}, \ldots, x_{n} \in U\left(x_{0}, r\right)$. Using the identity

$$
F\left(x_{n}\right)=F\left(x_{n}\right)-F\left(x_{n-1}\right)-P A\left(u_{n-1}, v_{n-1}\right)\left(x_{n}-x_{n-1}\right),
$$


(2) and (12) we get

$$
\left\|F\left(x_{n}\right)\right\| \leq c\left\|x_{n}-x_{n-1}\right\|^{1+d_{n}}
$$

and

$$
\left\|x_{n+1}-x_{n}\right\| \leq b \cdot c\left\|x_{n}-x_{n-1}\right\| \leq \cdots \leq h \cdot \eta^{1+\left(1+d_{n}\right)+\cdots+\left(1+d_{2}\right)+\cdots+\left(1+d_{n}\right)} .
$$

Hence,

$$
\left\|x_{n+1}-x_{n}\right\| \leq h^{q_{n}} \eta
$$

From the inequality

$$
\left\|x_{0}-x_{n+1}\right\| \leq \sum_{i=1}^{n+1}\left\|x_{i}-x_{i-1}\right\| \leq \sum_{i=0}^{n} h^{q_{i}} \eta \leq q_{0} \eta \leq r
$$

we deduce that $x_{n+1} \in U\left(x_{0}, r\right)$. From

$$
\left\|x_{n}-x_{n+p}\right\| \leq \sum_{i=n}^{n+p-1} h^{q_{i}} \eta \leq q_{n} \eta, \quad n \geq 0
$$

we get that the sequence $\left\{x_{n}\right\}, n \geq 0$ is a Cauchy sequence in a Banach space and as such it converges to some $x^{*}$. By taking $p \longrightarrow \infty$ we get (17) and $x^{*} \in U\left(x_{0}, r\right)$. Finally, by letting $n \longrightarrow \infty$ in (18) we get $F\left(x^{*}\right)=0$.

That completes the proof of the theorem.

Note that if it is Fréchet differentiable and

$$
\left\|F^{\prime}(x)-F^{\prime}(y)\right\| \leq c\|x-y\| \text { for all } \quad x, y \in \bar{U}\left(x_{0}, r\right)
$$

then we can set $d_{i}=1$ and $c=\frac{1}{2} \sup _{E}\left\|F^{\prime \prime}(x)\right\|$ in Theorem 4. Moreover, note that condition (20) has been used by various authors [1], [2], [4], [5] to prove convergence for Newton's method

$$
z_{n+1}=z_{n}-F\left(z_{n}\right)^{-1} F\left(z_{n}\right), \quad n \geq 0 .
$$

Furthermore, if the Fréchet derivative $F^{\prime}(x)$ of $F$ is Hölder continuous on $\bar{U}\left(x_{0}, r\right)$, that is

$$
\left\|F^{\prime}(x)-F^{\prime}(y)\right\| \leq c\|x-y\|^{\lambda}, \quad 0<\lambda<1, \quad x, y \in \bar{U}\left(x_{0}, r\right)
$$

then our Theorem 4 through (21) shows that the order of convergence of iteration $\left\{z_{n}\right\}$, $n \geq 0$ is $1+\lambda,[1]$, [2].

We now complete this paper with an application. For simplicity we take $P=I$.

IV. Applications. 
Let $E=\hat{E}=R^{2}$ and consider a system of two nonlinear equations

$$
\begin{aligned}
& q_{1}(s, t)=0 \\
& q_{2}(s, t)=0 .
\end{aligned}
$$

Then $x=(s, t), F(x)=\left(q_{1}(s, t), q_{2}(s, t)\right)$. Let

$$
\begin{gathered}
x_{i}=\left(s_{i}, t_{i}\right), \\
F\left(x_{i}\right)=\left(q_{1}\left(s_{i}, t_{i}\right), q_{2}\left(s_{i}, t_{i}\right)\right), \\
x_{i+1}-x_{i}=\Delta x_{i}=\left(s_{i+1}-s_{i}, t_{i+1}-t_{1}\right)=\left(\Delta s_{i}, \Delta t_{i}\right) .
\end{gathered}
$$

From (2) we get

$$
A\left(y_{n}, y_{n-1}\right) \triangle x_{n}=F\left(x_{n}\right)
$$

where

$$
A(u, v)=\left[\begin{array}{ll}
\frac{q_{1}\left(u_{1}, u_{2}\right)-q_{2}\left(v_{1}, v_{2}\right)}{u_{1}-v_{1}} & \frac{q_{1}\left(v_{1}, u_{2}\right)-q_{1}\left(v_{1}, v_{2}\right)}{u_{2}-v_{2}} \\
\frac{q_{2}\left(u_{1}, u_{2}\right)-q_{2}\left(v_{1}, u_{2}\right)}{u_{1}-v_{1}} & \frac{q_{2}\left(v_{1}, u_{2}\right)-q_{2}\left(v_{1}, v_{2}\right)}{u_{2}-v_{2}}
\end{array}\right]
$$

for $u=\left(u_{1}, u_{2}\right)$ and $v=\left(v_{1}, v_{2}\right)$.

In order to calculate $\Delta x_{n}=\Delta u_{n}, \Delta v_{n}$ ) we must solve (22) which is a system of algebraic equations. If the rest of the assumptions of Theorems 1 and 4 are satisfied then the conclusions apply.

\section{References}

[1] Argyros, I.K. The Secant method and fixed points of nonlinear operators. Monatshefie für Mathematik, 106, (1988), 85-94.

[2] — Concerning the approximate solutions of operator equations in Hilbert space under mild differentiability conditions. Tamkang J. Math., Vol. 19, 4 (1988).

[3] Baluev, A. On the method of Chaplygin. Dokl. Akad. Nauk. SSSR. 83, (1952), 781-784.

[4] Kantorovich, L.V. The method of successive approximation for functional equations. Acta Math. $71,(1939), 63-97$.

[5] Ortega, J.M. and Rheinboldt, W.C. Iterative solution of nonlinear equations in several variables. Academic Press, New York, (1970).

[6] Potra, F.A. Newton-like methods with monotone convergence for solving nonlinear operator equar tions. Nonlinear analysis theory methods and application. Vol. 11, 6, (1987), 697-717.

[7] Schmidt, J.W. and Leonhardt, H. Eingrenzung von Lösungen mit Hilfe der Regula falsi. Computing, 6 (1970), 318-329.

[8] Vanderfraft, J.S. Newton's method for convex operators in partially ordered spaces. SIAM J. Numer. Anal. 4, (1967), 402-432.

Department of Mathematical Sciences, New Mexico State University, Las Cruces, NM 88003. 